\title{
Development of PET Fabrics Containing N-halamine Compounds with Durable Antibacterial Property
}

\author{
Fang Ding ${ }^{1}$, Shumin Zhang ${ }^{1}$, Xuehong Ren ${ }^{1 *}$, and Tung-Shi Huang ${ }^{2}$ \\ ${ }^{1}$ Key Laboratory of Eco-textiles of Ministry of Education, College of Textile Science and Engineering, Jiangnan University, \\ Wuxi, Jiangsu 214122, China \\ ${ }^{2}$ Department of Poultry Science, Auburn University, Auburn, Alabama 36849, USA
}

(Received May 1, 2021; Revised June 10, 2021; Accepted June 15, 2021)

\begin{abstract}
Antibacterial textile materials are widely used in daily life, but most are disposable products with poor antibacterial durability. N-halamine can rapidly inactivate microorganisms, has good stability, and shows great potential applications in antibacterial fabrics. In this study, an N-halamine monomer precursor was synthesized and treated onto PET fabrics. The treated PET fabrics were rendered antibacterial functionality after chlorination, and exhibited good antibacterial properties with inactivation rate of $100.0 \%$ for both E. coli $\mathrm{O} 157: \mathrm{H} 7$ and $S$. aureus. After 50 wash cycles, the chlorinated treated PET fabrics could maintain $80.0 \%$ antibacterial efficacy, demonstrating durable antibacterial properties. Storage stability and UV irradiation tests showed that the treated PET fabrics had remarkable regenerable properties. The reduction of the breaking strength was within $12 \%$ after treatment, which is in a satisfying range in antimicrobial finishing.
\end{abstract}

Keywords: PET fabrics, N-halamine, Antibacterial, Washing stability, Durability

\section{Introduction}

Emerging infectious diseases and their negative impact on public health have become the forefront of global safety concerns [1]. Skin, a protective barrier and a first line of defense, does not protect against all viruses, potentially spreading disease, leading to death [2]. Asymptomatic bacterial infections are one of the biggest health risks in daily life $[3,4]$. Furthermore, fabrics in contact with human body are ideal carriers for the growth of microorganisms and are easily contaminated with pathogenic viruses threatening human health. Antibacterial textiles can prevent the growth of microorganisms and reduce the occurrence of diseases effectively. As one of the most widely used fabrics, polyethylene terephthalate (PET) fabric exhibits excellent physical properties, easy washing, high dimensional stability and low cost [5]. Imparting PET fabrics with antibacterial properties would potentially protect public safety.

To endow fabrics with antibacterial properties, either quaternary ammonium salts or metal ions have been extensively adopted [6]. However, considering the issues of effectiveness, reusability, safety and durability, there are still many obstacles to overcome. As the most commonly used antibacterial compound, the antibacterial efficiency of quaternary ammonium salt could not meet the demand [7, 8]. Antimicrobial textiles with inorganic metal particles, such as silver ions, focus on enhancing the adherence of metal particles onto fabrics in order to prevent leaching into environment and causing harm [9-11]. In addition, natural antimicrobial agents with environmental friendliness and nontoxicity were also frequently incorporated into polymeric materials $[12,13]$ but have some disadvantages such as high

*Corresponding author: xhren@jiangnan.edu.cn extraction cost, low sterilization efficiency and weak thermal stability [14]. As synthetic antimicrobial agents, N-halamine compounds have been applied in medical dressings $[15,16]$, filtration materials $[17,18]$ and textiles $[19,20]$ due to their excellent biocompatibility and high antibacterial activity [21-23]. Conveniently, after chlorination by household bleach solution, the N-H bond in N-halamine can be converted to the $\mathrm{N}-\mathrm{Cl}$ bond, which has high antimicrobial efficiencies and rechargeable properties [24] and the level of chlorine loading represents the antibacterial activity to a certain extent $[25,26]$. The oxidative chlorine is controlled and protected by the dissociation constant of the N-halogen group [27], maintaining stability.

To broaden the application of N-halamine, synthesis and modification with structural functionality are worthy of exploration. The frequently used method is to modify $\mathrm{N}$ halamine compounds with functional groups, and then apply them into hydrogel, aerogel nanofibrous membranes and coating them onto fabrics for specific applications [28-30]. For instance, 5,5-dimethylhydantoin is the most widely used starting-material in the preparation of hydantoin-containing $\mathrm{N}$-halamines due to its high reactivity via a substitution reaction [31]. The preparation of antibacterial PET fabrics by N-halamine compounds based on 5,5-dimethylhydantoin has been reported [32-34]. However, the high efficiency of antibacterial PET fabrics needs to be further improved. In addition, the washing stability of antibacterial fabrics needs to be addressed [35,36]. 3-(3'-Acrylicacidpropylester)-5,5dimethylhydantoin (APDMH) has been synthesized and reported to treat cotton fabrics by electron beam irradiation and coat onto graphene oxide nanosheets by in-situ polymerization [37,38]. The existence of APDMH enables the substrates to obtain excellent antibacterial properties. It has been reported that the hydrophobicity of the substrate 


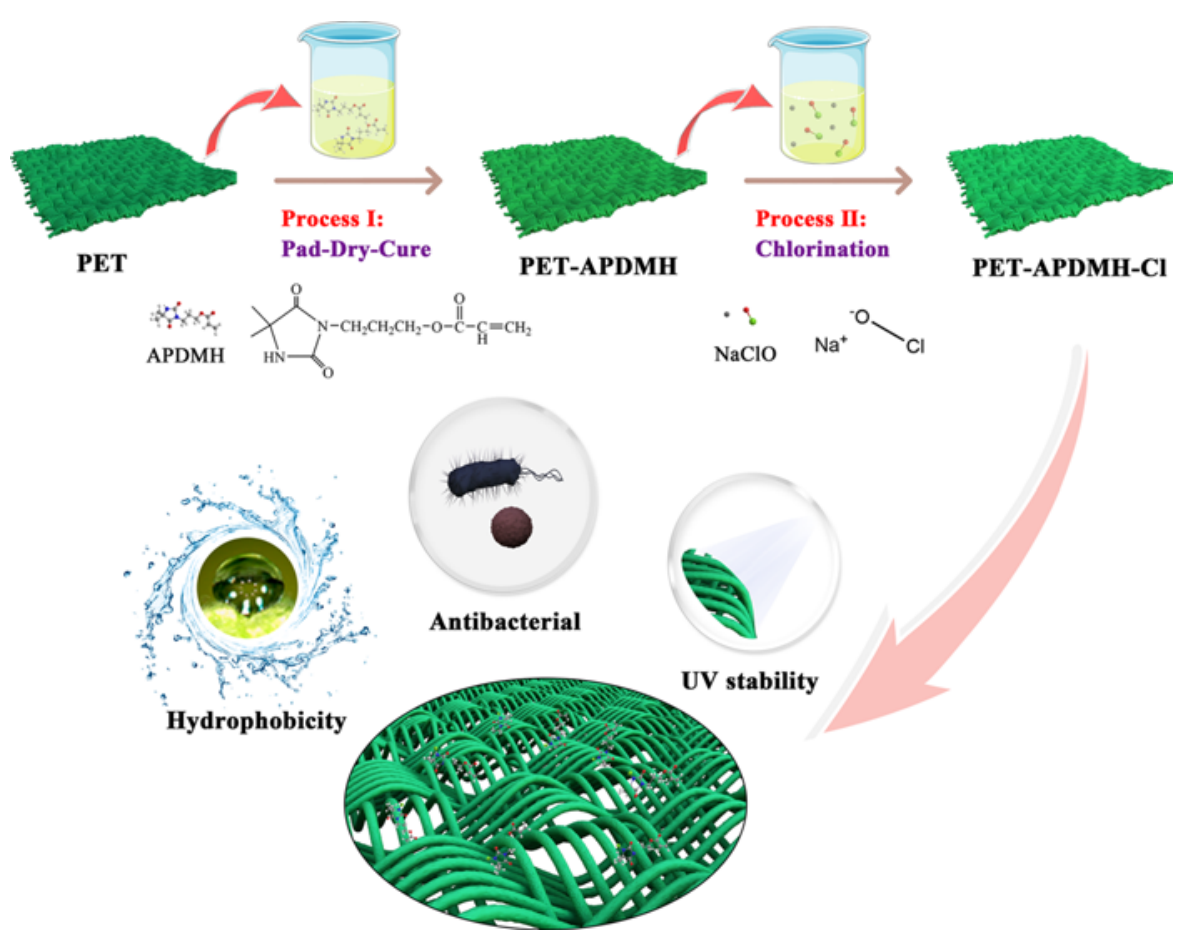

Scheme 1. Schematic illustration of the preparation of antibacterial PET fabrics.

based on N-halamine has notable impacts on the antibacterial performance and the stability of the N-halamine structure [39]. An alternative strategy is needed to be developed to obtain durable antibacterial PET fabrics since PET fabrics lack functional groups as compared with cotton fabrics.

In this work, APDMH was first used for the preparation of antibacterial PET fabrics for excellent antibacterial activity and improving durability. An alkali process was not necessary before antibacterial treatment of PET fabrics. APDMH was coated onto the surface of PET fabrics via a pad-dry-cure approach with the help of azobis (isobutyronitrile). The stability and hydrophobicity of PET fabrics can provide long-lasting antibacterial functions of $\mathrm{N}$-halamine. Moreover, the effect of APDMH on the mechanical, thermal, durability and antibacterial properties of the treated fabrics were investigated. The treated PET fabrics can be used as protective clothing in the field of public health.

\section{Experimental}

\section{Materials}

PET fabrics $\left(24 \times 13\right.$ yarn/inch, $\left.356 \mathrm{~g} / \mathrm{m}^{2}\right)$ were purchased from Nuosi Cleaning Products Co., Ltd. Acryloyl chloride, azobis (isobutyronitrile) (AIBN) and 3-chloro-1-propanol were obtained from J\&K Scientific Co., Ltd. 5,5Dimethylhydantoin was provided from Hebei Yaguang Fine Chemical Co., Ltd. The bacteria used in the test were $S$. aureus (ATCC 6538) and E. coli O157:H7 (ATCC 43895) (American Type Culture Collection, Rockville, MD). Tetrahydrofuran (THF), triethylamine (TEA), sodium hydroxide $(\mathrm{NaOH})$, acryloyl chloride, acetone, sulfuric acid $\left(\mathrm{H}_{2} \mathrm{SO}_{4}, 98 \%\right)$, sodium thiosulfate $\left(\mathrm{Na}_{2} \mathrm{~S}_{2} \mathrm{O}_{3}\right)$, potassium iodide $(\mathrm{KI})$, sodium hypochlorite $(\mathrm{NaClO})$, glutaraldehyde and other chemicals were purchased from Sinopharm Chemical Reagent Co., Ltd. All of the chemical reagents<smiles>CC1(C)NC(=O)NC1=O</smiles><smiles>CC1(C)NC(=O)N(CCCO)C1=O</smiles><smiles>C=CC(=O)Cl</smiles><smiles>C#CC1(C)NC(=O)N(CCCOC(=O)C=C)C1=O</smiles>

Figure 1. Synthesis of APDMH. 
were used without further purification.

\section{Preparation of APDMH}

APDMH was synthesized by following the method reported in the literature [37,38] and Figure 1 shows the synthetic route of APDMH. Briefly, $0.1 \mathrm{~mol}$ of 5,5dimethylhydantoin and $\mathrm{NaOH}$ with equal molar volume $(0.1 \mathrm{~mol})$ were dissolved in $100 \mathrm{ml}$ deionized water and stirred for $15 \mathrm{~min}$. Then $0.1 \mathrm{~mol}$ of 3-chloro-1-propanol was added to the above mixture and stirred continuously at $100{ }^{\circ} \mathrm{C}$ for $10 \mathrm{~h}$. The product purified by acetone was dissolved in THF, and 0.1 mol TEA and $0.1 \mathrm{~mol}$ acryloyl chloride were added dropwise and the mixture was stirred at room temperature for $20 \mathrm{~h}$. After the reaction, the byproduct of triethylamine hydrochloride was removed by filtration, and THF was removed by decompression distillation. Then, the product was washed repeatedly with THF and acetone three times for $5 \mathrm{~min}$. Finally, the solvent was removed by vacuum distillation, and the target product (yield: $80 \%$ ) was obtained.

\section{Preparation of the Treated PET Fabrics}

PET fabrics were washed with detergent to remove impurities from the surface before experimentation. The treating solution was prepared by dissolving $1 \%$ (weight to the fabrics) APDMH and $2 \%$ (weight to APDMH) AIBN in a mixture of $70 \mathrm{~m} l$ deionized water and $30 \mathrm{~m} l$ ethanol. The PET fabrics were dipped into the treating solution for $30 \mathrm{~min}$ and padded ( $100 \%$ wet pick-up), dried at $60^{\circ} \mathrm{C}$ for $3 \mathrm{~min}$, and baked at $150^{\circ} \mathrm{C}$ for $5 \mathrm{~min}$. The unreacted monomers and homopolymers on the surface of fabrics were removed with ethanol, and the washed fabrics were dried at $60^{\circ} \mathrm{C}$ to constant weight and denoted as PET-APDMH.

\section{Chlorination and Analytical Titration}

In order to convert the amide bond on the treated fabrics into an N-halamine structure with rapid and efficient antibacterial activity, the samples were added into $10 \%$ $\mathrm{NaClO}$ solution ( $\mathrm{pH}=7$, adjusted by diluted sulfuric acid) at a 1:100 bath ratio and stirred at room temperature for $1 \mathrm{~h}$. The chlorinated fabrics were thoroughly washed with deionized water and then dried at $45^{\circ} \mathrm{C}$ for $1 \mathrm{~h}$ to remove unbounded chlorine on the surface of the fabrics. The chlorinated PET fabrics were denoted as PET-Cl and PETAPDMH-Cl.

Chlorine loading of the active chlorine content of samples was determined by iodometric titration, which is widely used in the preliminary determination of the antibacterial properties of fabrics [40,41]. Briefly, $0.15 \mathrm{~g}$ of the treated fabrics were cut into pieces and then added into a beaker with $15 \mathrm{ml}$ deionized water and $0.1 \mathrm{~g} \mathrm{KI}$. The mixture was stirred for $1 \mathrm{~h}$ to form iodine, and 5 drops of $1 \mathrm{wt} \%$ starch solution were added. During titration with $\mathrm{Na}_{2} \mathrm{~S}_{2} \mathrm{O}_{3}$ solution $(0.01 \mathrm{~N})$, the iodine was reduced to iodide ions changing to colorless at the titration endpoint. The oxidative $\mathrm{Cl}^{+} \mathrm{wt} \%$ in the sample was calculated according to the equation below (equation (1)).

$$
\mathrm{Cl}^{+}(\mathrm{wt} \%)=\frac{N \times\left(V_{1}-V_{0}\right) \times 35.45}{2 m} \times 100
$$

where, $\mathrm{N}, \mathrm{V}_{0}$ and $\mathrm{V}_{1}$ represent the normality (Equiv/L), the volumes (L) to titrate blank and the sample of used $\mathrm{Na}_{2} \mathrm{~S}_{2} \mathrm{O}_{3}$ titrant, respectively, and $\mathrm{m}$ is the weight $(\mathrm{g}$ ) of the tested samples.

\section{Antibacterial Evaluation of the Treated PET Fabrics}

A modified AATCC 100-2004 Test Method (termed as a sandwich method) was used to evaluate the antibacterial activities of samples against both Gram-positive $S$. aureus (ATCC 6538) and Gram-negative E. coli O157:H7 (ATCC 43895), which was a representative test for the evaluation of antibacterial efficiency $[42,43]$. An aliquot of $25 \mu l$ bacteria suspension was added to sandwich of the stacked two samples $(2.54 \times 2.54 \mathrm{~cm})$. The residual active chlorine on the tested samples was quenched with $5 \mathrm{~m} l$ of sodium thiosulfate $(0.02 \mathrm{~N})$ after contact time of $1,5,10$, and $30 \mathrm{~min}$. Then the quenched bacterial suspensions were diluted with $0.03 \mathrm{~mol} / \mathrm{L}$ phosphate buffer solution (PBS, $\mathrm{pH}$ 7.4) and plated onto Trypticase soy agar plate and incubated at $37^{\circ} \mathrm{C}$ for $24 \mathrm{~h}$. The number of bacterial colonies on the plate was counted for biocidal efficacy analysis of each tested sample.

\section{Bacteria Observation}

The bacteria morphologies were observed according to the previous reports [44-46], samples quenched from the antibacterial test were immersed in PBS solution containing $2.5 \mathrm{wt} \%$ of the glutaraldehyde at $4{ }^{\circ} \mathrm{C}$ for one night to immobilize the bacterial cells on the samples. Thereafter, the samples were washed with PBS solution and bacterial cells were dehydrated by soaking the samples in a series of graded alcohol-PBS solutions (ethanol concentration of $25 \%, 50 \%, 75 \%, 85 \%, 90 \%, 95 \%$, and $100 \%$ ). Finally, the samples were dried in a vacuum oven at $45{ }^{\circ} \mathrm{C}$ for $24 \mathrm{~h}$ for SEM observation.

\section{Contact Angle Measurement of the Treated PET Fabrics}

Statistic water contact angles (WCA) of fabrics were evaluated by water contact angle using a digital microscope contact angle tester (26,700-300, Instrument \& Equipment Specialties Inc., America). Drops of $10 \mu l$ of distilled water were dropped on the flat surface of fabrics, and the contact angle was measured after $1 \mathrm{~min}$. Each sample was tested at 5 different spots and the mean of each sample was used for analysis.

\section{Storage Stability}

The storage stability of PET-APDMH-Cl was tested according to the method reported by the literature [47,48]. In 
brief, PET-APDMH-Cl was prepared and put into a selfsealed bag at ambient temperature in the dark. After a period of time $(0,5,10,15,20,25$, and $30 \mathrm{~d})$, the chlorine contents of the samples were measured by titration method.

\section{UV Light Stability}

The UV light stability of PET-APDMH-Cl was investigated according to ASTMD4587 standards with an Accelerated Weathering Tester (The Q-LAB Company, USA). Each tested sample was exposed horizontally to the UV light $\left(315-400 \mathrm{~nm}, 0.89 \mathrm{~W} / \mathrm{m}^{2}, 60^{\circ} \mathrm{C}\right)$ in a UV chamber for a series of exposure time intervals. After the exposure, the samples were removed from the UV chamber and the remaining chlorine loading was measured.

\section{Washing Stability}

The washing test of the samples was carried out by repeated standard washing according to the standard AATCC 61-1996 Test Method. Six PET-APDMH-Cl samples $(2.54 \mathrm{~cm} \times 5.08 \mathrm{~cm})$ and three PET-APDMH samples $(2.54 \mathrm{~cm} \times 5.08 \mathrm{~cm})$ were placed in a stainless steel canister with 50 stainless steel balls and $150 \mathrm{~m} l$ AATCC detergent water solution and rotated with $42 \mathrm{rpm}$ at $49^{\circ} \mathrm{C}$. One washing cycle is $45 \mathrm{~min}$ and equivalent to 5 machine washings. After washed 5, 10, 25, and 50 machine launderings, the fabrics were rinsed with distilled water and dried. By following the previous procedure $[49,50]$, three PET-APDMH-Cl samples and three PET-APDMH samples after washing were chlorinated according to the chlorination method and were denoted as PET-APDMH-Cl-Wn-Cl ( $\mathrm{n}$ is the number of machine launderings) and PET-APDMH-Wn$\mathrm{Cl}$. Another three PET-APDMH-Cl samples after washing without further chlorination were denoted as PET-APDMH$\mathrm{Cl}-\mathrm{Wn}$. Then all the 9 samples were titrated, and the average chlorine content was recorded, respectively.

\section{Instruments and Characterization}

Fourier transform infrared (FT-IR) spectra were recorded by a NICOLET Is10 FT-IR spectrometer (Nicolet Instrument Corporation, USA). The surface morphology of samples was characterized by a TM3030 scanning electron microscope (SEM) (Hitachi, Japan) and a SU-1510 scanning electron microscope (Hitachi, Japan) was used to illustrate the microstructure of bacteria. A SU 1510 energy dispersive $\mathrm{X}$-ray spectrometer (EDX, Hitachi, Japan) was used to analyze the surface elemental composition of samples. To verify the chemical state of elements on the surface of samples, X-ray photoelectron spectroscopy (XPS) was carried out on an ESCALAB 250Xi (Thermo Fisher, USA). A Q500 thermogravimetric analyzer (TGA, TA Instruments, USA) was used to determine the thermal stability of fabrics with temperature ranging from $50{ }^{\circ} \mathrm{C}$ to $600{ }^{\circ} \mathrm{C}$ in a nitrogen

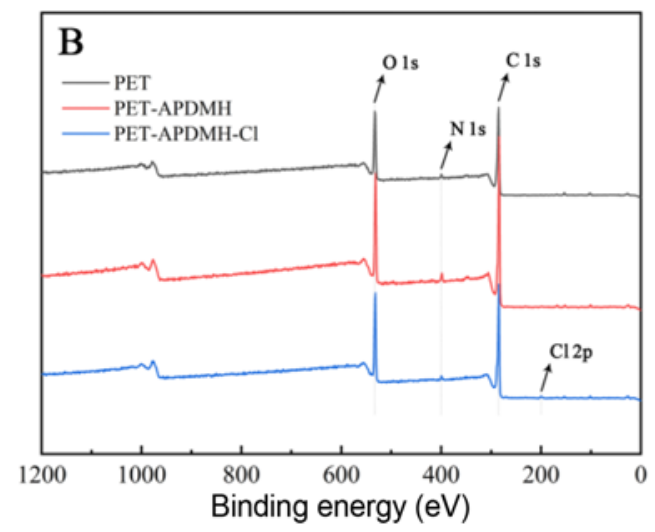

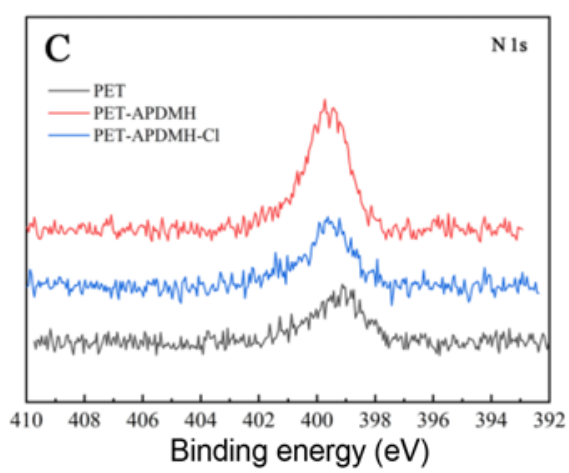
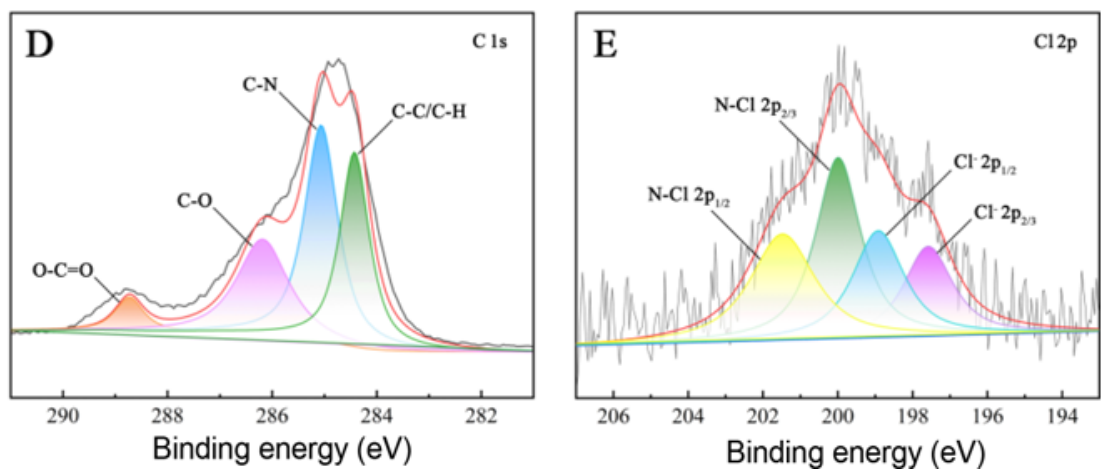

Figure 2. (A) FT-IR spectra of PET, APDMH, PET-APDMH and PET-APDMH-Cl, (B) XPS spectra of PET, PET-APDMH and PETAPDMH-Cl, (C) N 1s spectrum of PET, PET-APDMH and PET-APDMH-Cl, (D) C 1s, and (E) Cl 2p core-level spectrum of PET-APDMH-Cl. 
atmosphere with a linear heating rate of $10^{\circ} \mathrm{C} / \mathrm{min}$. Breaking strengths of samples were assessed by an electronic fabrics strength tester (HD026 N, Hongda Experimental Instrument Company, China) with a loading rate of $100 \mathrm{~mm} / \mathrm{min}$ at room temperature according to $\mathrm{GB} / \mathrm{T}$ 3923.1-2013 standard method. The samples $(15 \mathrm{~cm} \times 5 \mathrm{~cm})$ were tested in quintuplicate and the results were averaged.

\section{Results and Discussion}

\section{Characterization and Morphology}

FT-IR and XPS were used to determine the chemical composition and chemical binding state of the treated PET fabrics. After the treatment with APDMH, new absorption peaks appeared at $1712 \mathrm{~cm}^{-1}$ and $1408 \mathrm{~cm}^{-1}$ in the curves of PET-APDMH and PET-APDMH-Cl (Figure 2A), which were attributed to the stretching mode of carbonyl group $(-\mathrm{C}=\mathrm{O})[51,52]$ and the bending vibrations of alkyl groups in the N-halamine structure [38], respectively. XPS spectra of PET, PET-PAPDMH, PET-APDMH-Cl were performed, and the results are shown in Figure 2B-E. Small amount of nitrogen were detected in PET, which might originate from the dye on it. The peaks of PET-APDMH and PETAPDMH-Cl were significantly enhanced at $399.08 \mathrm{eV}$, which was the binding energy of $\mathrm{N} \mathrm{1s}$, indicating that APDMH has been treated onto the surface of the PET fabrics. Compared with PET-APDMH, Cl $2 \mathrm{p}$ in PETAPDMH-Cl appeared at $200.8 \mathrm{eV}$, which was attributed to the formation of the $\mathrm{N}-\mathrm{Cl}$ bond after chlorination $[53,54]$. The presence of the $\mathrm{Cl}$ element suggested that $\mathrm{N}-\mathrm{H}$ bond was successfully shifted into N-C bond on the surface of the treated PET fabrics.

The surface morphologies of PET and the treated samples were explored by SEM visually. According to the image in Figure $3 \mathrm{~A}$, the surface of PET fabrics was relatively smooth. In contrast, after treatment with APDMH, PET-APDMH with layer attached was apparently observed, as shown in Figure 3B. The surface of PET-APDMH shows more sediments than the untreated sample, which was due to the treatment with APDMH. Figure 3C further shows uniform
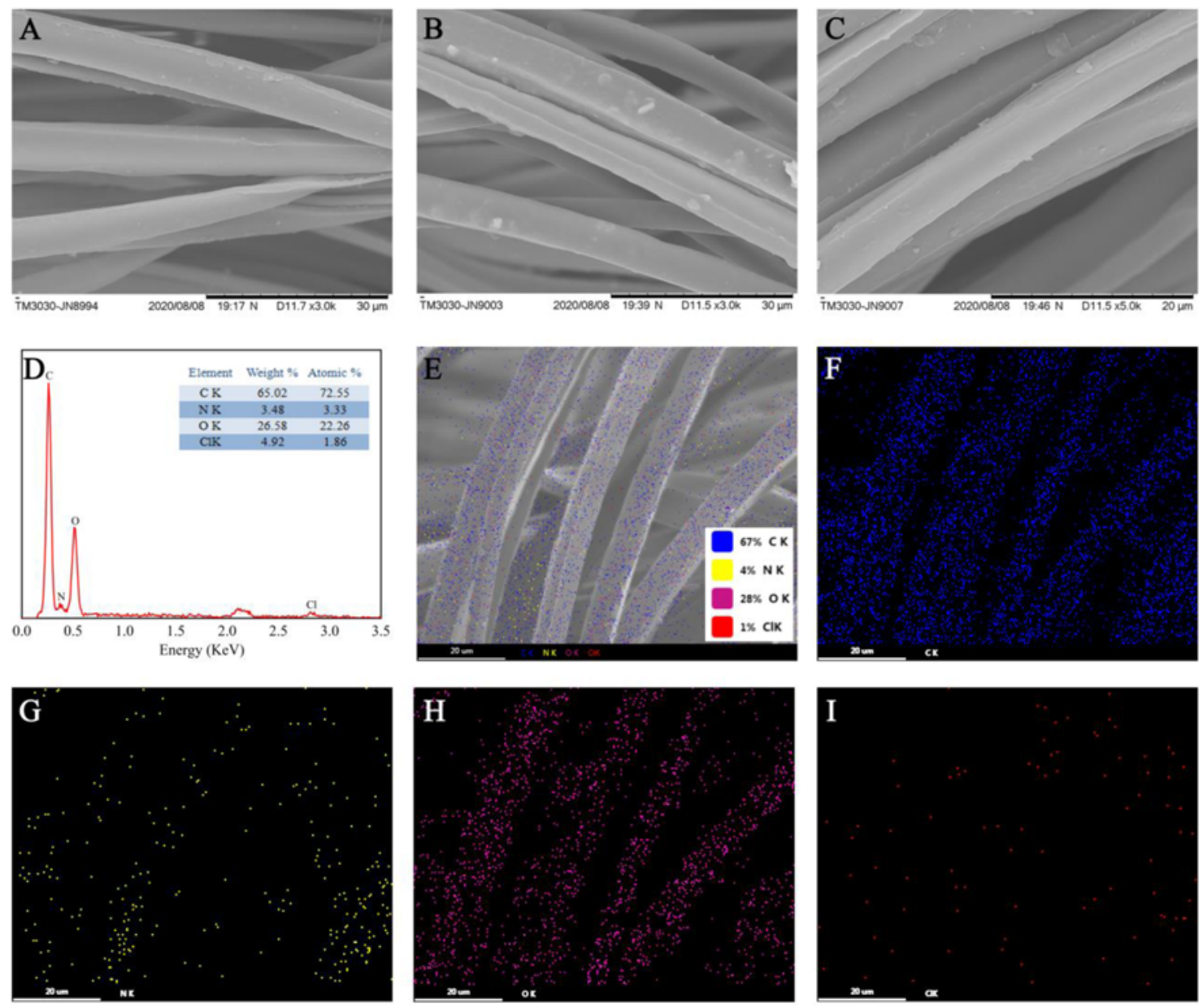

Figure 3. SEM micrographs of (A) PET, (B) PET-APDMH, (C) PET-APDMH-Cl and EDS analysis of PET-APDMH-Cl (D) EDS graph of the sample, (E) the total point distribution of $\mathrm{C}, \mathrm{N}, \mathrm{O}$ and $\mathrm{Cl}$ elements, $(\mathrm{F})$ the point distribution of $\mathrm{C}$ element, $(\mathrm{G})$ the point distribution of $\mathrm{N}$ element, $(\mathrm{H})$ the point distribution of $\mathrm{O}$ element, and $(\mathrm{I})$ the point distribution of $\mathrm{Cl}$ element. 


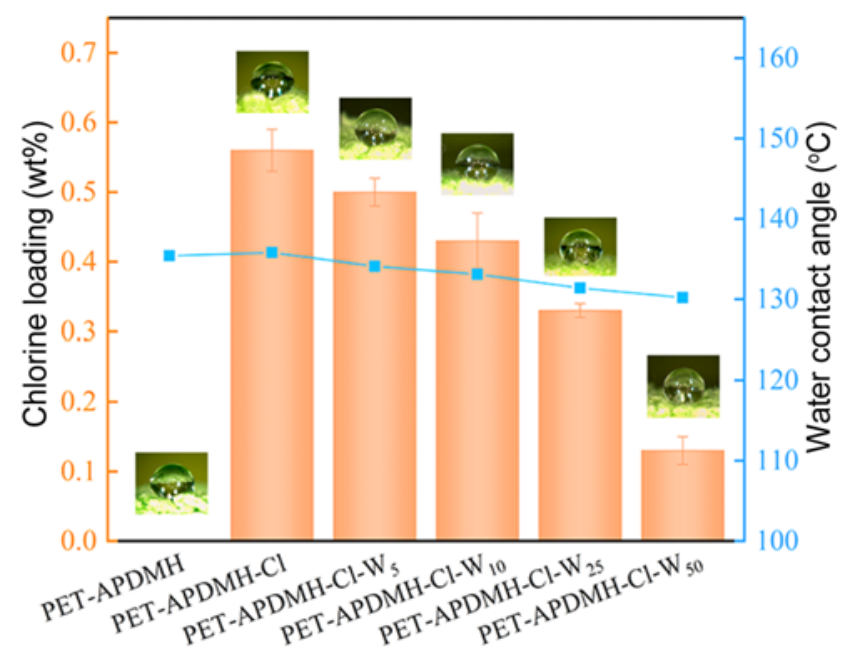

Figure 4. Chlorine loadings and water contact angles of samples. treatment after chlorination, and no visible change was observed.

The chemical composition also affects the antibacterial properties of the treated PET fabrics, and EDS was used to analyze the distribution of surface elements of PETAPDMH-Cl. A new peak of chlorine appeared at $2.8 \mathrm{KeV}$ as illustrated in Figure 3D, with a weight percentage of $4.92 \%$. Images of the elements distribution on PET-APDMH-Cl are displayed in Figure 3E-I. The presence of chlorine indicates that APDMH was treated onto PET fabrics and chlorinated, and the $\mathrm{Cl}$ element was distributed uniformly.

\section{Hydrophobicity}

The hydrophilicity and hydrophobicity of fabrics are also important factors affecting the antibacterial properties. The combination of antibacterial and hydrophobicity in fabrics is one of the research focuses and exhibits potential applications
A

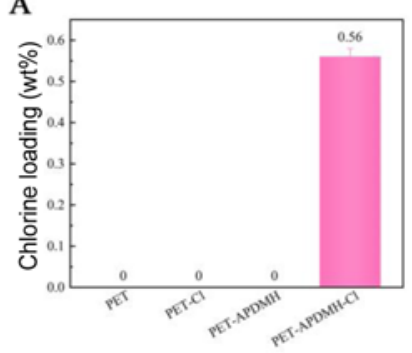

B

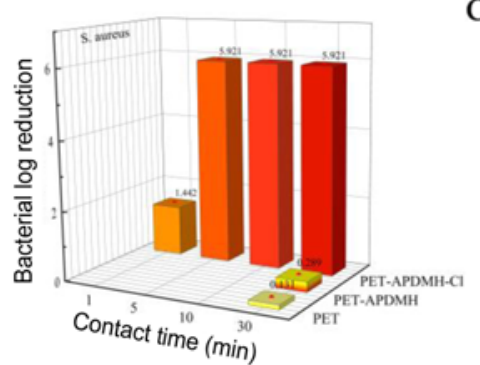

$\mathrm{C}$

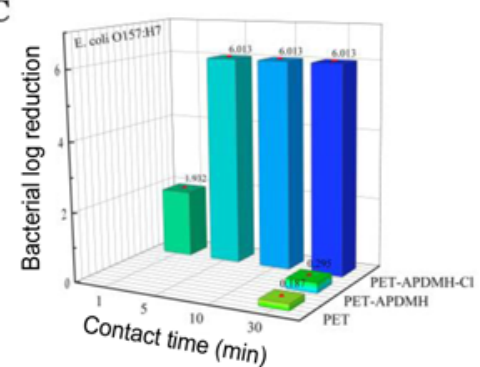

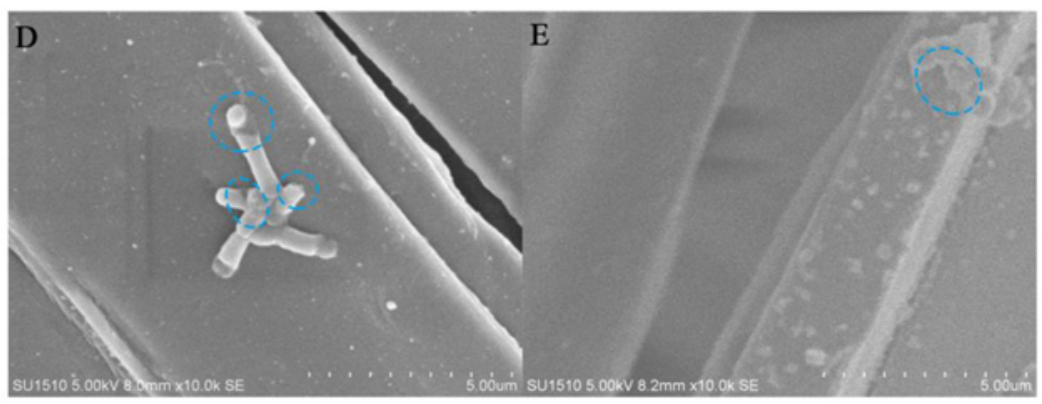

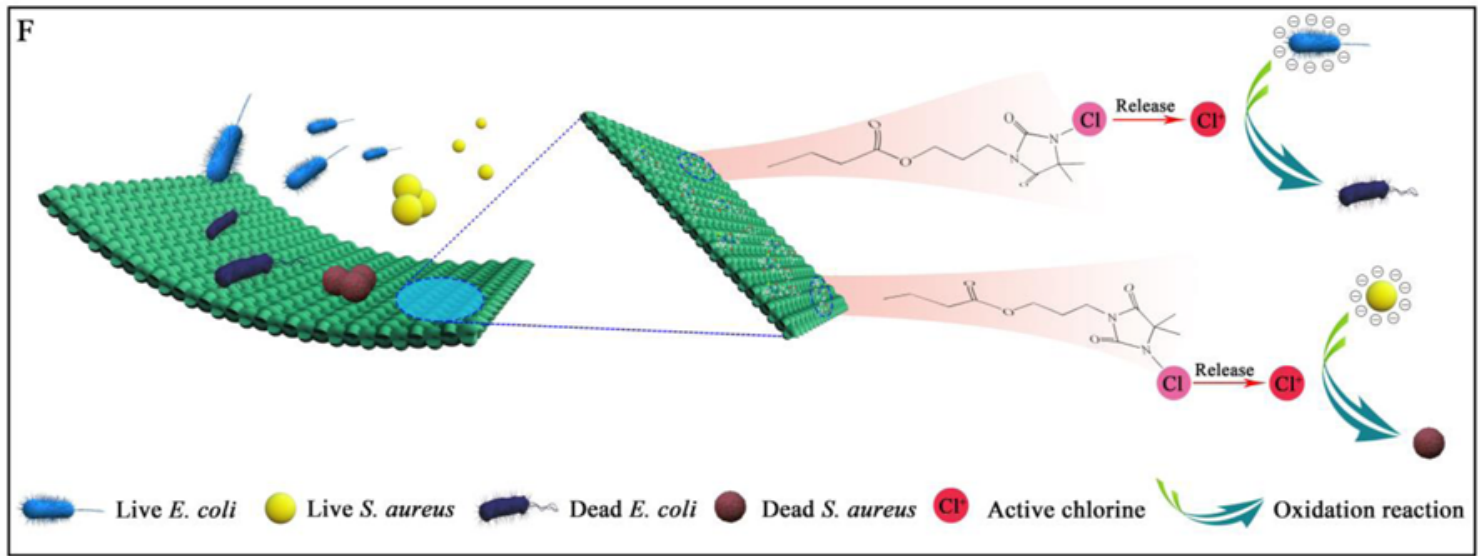

Figure 5. (A) The amount of active chlorine in different samples. Biocidal efficacy of different samples against (B) $S$. aureus and (C) E. coli O157:H7. SEM images of (D) E. coli O157:H7 and (E) S. aureus with PET-APDMH-Cl treatment. (F) Schematic illustration of the antibacterial process. 
in the industry [55-57]. Figure 4 depicts the water contact angles and chlorine content of different fabrics. The fabrics became hydrophobic after treated with APDMH with WCA of $135.4^{\circ}$. A slight change in WCA values was observed after chlorination and washing. The WCA values of PETAPDMH-Cl, PET-APDMH-Cl-W5, PET-APDMH-Cl-W10, PET-APDMH-Cl-W25 and PET-APDMH-Cl-W50 were $135.8^{\circ}, 134.1^{\circ}, 133.1^{\circ}, 131.4^{\circ}$ and $130.2^{\circ}$, respectively, which did not show a correlation with the chlorine content of fabrics. Therefore, the halogenation of PET-APDMH shows no impact on the hydrophobicity. The hydrophobicity of the fabrics reduces the contact between the fabrics and bacteria, which is beneficial to the sterile application of the fabrics.

\section{Antibacterial Efficacy}

The biocidal efficacies of PET, PET-APDMH and PETAPDMH-Cl were performed against $S$. aureus and E. coli O157:H7 at populations of $8.33 \times 10^{5}(5.921 \operatorname{logs})$ and $1.03 \times 10^{6}(6.013 \operatorname{logs}) \mathrm{CFU} / \mathrm{sample}$, respectively. The results of antibacterial testing are shown in Figure 5. Figure 5A shows that no active chlorine was detected in PET-Cl indicating that the PET was unchlorinated and did not contribute to the antibacterial function in the subsequent test. As we can see in Figure 5B and C, PET and PET-APDMH showed small reductions of both $S$. aureus and E. coli O157:H7 due to the adhesion of bacteria to fabrics instead of inactivation [58], indicating that the majority of bacteria were alive. In general, hydrophobic surfaces can cause a decrease of antibacterial efficacy due to the reduction of contact area between fabrics and bacteria [35]. The bactericidal effect on PET-APDMH was higher than that on PET, indicating that APDMH enhanced the affinity of fabrics to bacteria, which have a positive effect on biocidal effectiveness by increasing contact between the treated fabrics and bacteria. PET-APDMH-Cl with 0.56 wt \% oxidative chlorine could inactivate $1.442 \log S$. aureus and $1.932 \log E$. coli $\mathrm{O} 157: \mathrm{H} 7$ within $1 \mathrm{~min}$, respectively, and completely inactivated inoculated $S$. aureus and E. coli O157:H7 within 5 min of contact time, which is excellent for an antimicrobial application [59,60] The grafting and chlorination of APDMH enhanced the antibacterial properties of PET fabrics significantly. PET-APDMH-Cl showed highly effective antibacterial function and could kill all inoculated E. coli $\mathrm{O} 157: \mathrm{H} 7$ and S. aureus in a short period of time. The antibacterial activity was mainly derived from the contact disinfection of N-halamine compounds [61].

The morphologies of E. coli $\mathrm{O} 157: \mathrm{H} 7$ and S. aureus on PET-APDMH-Cl were observed by SEM images with $1000 X$ magnification to further investigate the antibacterial activity of the samples (Figure 5D and 5E). Normally, E. coli O157:H7 and S. aureus are rod-shaped and round-shaped, respectively, with smooth surfaces and plump colonies [62, 63]. The shape of E. coli $\mathrm{O} 157: \mathrm{H} 7$ was damaged with cellular deformation after contact with PET-APDMH-Cl, while intracellular contents were leaked out in S. aureus, but the cells were kept relatively intact due to the thicker cell walls. Upon exposure to the fabrics, bacteria first contacted N-halamine on the surface of PET-APDMH-Cl. With the
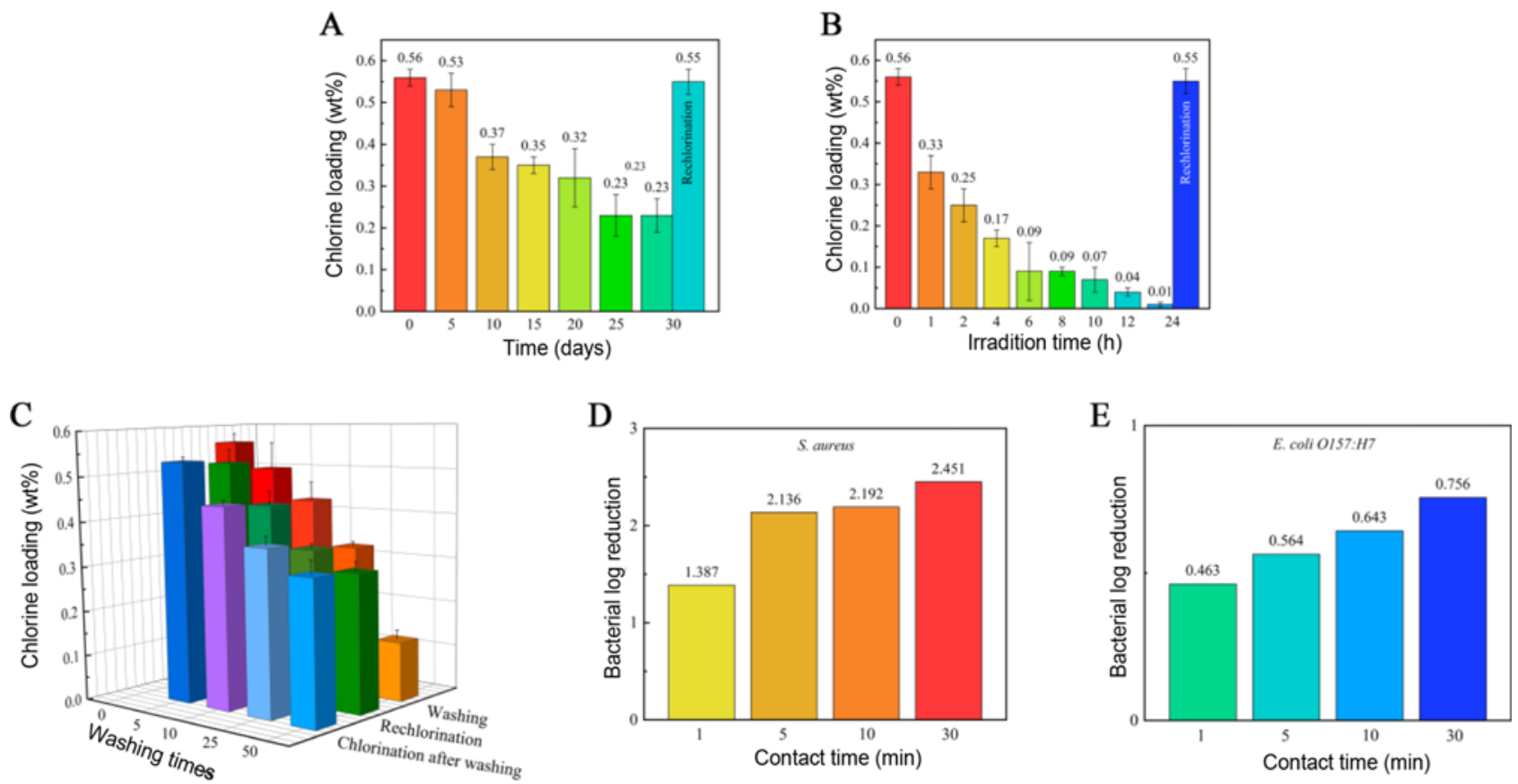

Figure 6. (A) Storage stability of PET-APDMH-Cl. (B) UV Stability of PET-APDMH-Cl. (C) Active chlorine in different washed samples. Biocidal Results of 50 washed PET-APDMH-Cl against (D) S. aureus and (E) E. coli O157:H7. 
extension of the contact time, the oxidative chlorine ions $(\mathrm{Cl}+)$ generated from $\mathrm{N}-\mathrm{Cl}$, bound with the negative charge on the surface of the bacteria causing irreversible change of bacteria cell membrane and leakage of cytoplasmic constituents killing the microorganisms (Figure 5F).

\section{Stability Assessment}

The stability performance stability of the treated fabrics has a profound influence on its application, and the chlorine stability of PET-APDMH-Cl after dark storage, UV light irradiation and washing was measured. Figure $6 \mathrm{~A}$ shows the decrease in the chlorine loading over different storage times when the samples were kept in the dark. After 30 days, $0.23 \mathrm{wt} \%$ oxidative chlorine of PET-APDMH-Cl was retained, which is still sufficient to inactivate bacteria [64]. The high chlorine content retained was due to the low dissociation constant of amide groups. In contrast, the active chlorine content decreased rapidly with the extension of UV irradiation time, and all the active chlorine content lost after $24 \mathrm{~h}$ exposure (Figure 6B). After re-chlorination, more than $98 \%$ of the chlorine loading of both stored and UVirradiated samples were regained, which indicated that the bonds between the APDMH and PET fabrics were stable under storage and UV light. The loss of chlorine was mainly due to the decomposition of $\mathrm{N}-\mathrm{Cl}$ bonds rather than the departure of APDMH from fabrics, which could be almost completely restored by the re-chlorination reaction. Overall, the rechargeability and good storage stability of chlorine greatly expand the application of the treated PET fabrics.

The washing stability of the treated fabrics is an important measure of performance for its application as well. Figure 6C-E show that the chlorine loadings of PET-APDMH-Cl after $5,10,25$, and 50 cycles washing were $0.50,0.43,0.33$, and 0.13 wt $\%$, respectively. After 50 washing cycles, about $23.2 \%$ of oxidative chlorine was retained, and $0.756 \mathrm{log}$ of E. coli $\mathrm{O} 157: \mathrm{H} 7$ and $2.451 \log$ of $S$. aureus were killed, indicating antibacterial property was maintained. The chlorine contents of PET-APDMH-Cl- $\mathrm{W}_{50}-\mathrm{Cl}$ and PETAPDMH- $\mathrm{W}_{50}-\mathrm{Cl}$ were 0.30 and $0.31 \mathrm{wt} \%$, respectively, and $53.6 \%$ and $55.4 \%$ of chlorine were restored after rechlorination. The retention of chlorine content indicated that
PET-APDMH has good washing stability and can be reused for many times. In general, the good storage stability, UV stability and washing stability shows that PET-APDMH fabrics have practical application.

\section{Thermal Behaviors and Mechanical Properties}

To study the influence of APDMH on the thermal behaviors and mechanical properties of PET fabrics, thermal analysis in nitrogen atmosphere and breaking strength of different samples were measured. Figure 7A and B show that there were no remarkable differences in the decomposition temperature and maximum decomposition rate of PET and PET-APDMH, indicating that the treatment of APDMH on PET fabrics did not significantly affect the thermal performance. The transformation and decomposition of $\mathrm{N}$ $\mathrm{Cl}$ bonds did not accelerate the thermal decomposition of fabrics, indicating that the thermal behavior of the fabrics would be not affected after treatment with APDMH. Figure 7C shows the breaking strengths of different fabrics, and the breaking strengths of fabrics in warp and weft directions, which decreased by $7.74 \%$ and $11.21 \%$ after chlorination, respectively. The coating of the fabrics and the chlorination of N-H bonds had little effect on the strength of the fabrics. The thermal properties and strength of the treated fabrics were preserved, which is due to the small and efficient loading of APDMH on the surface of PET fabrics.

\section{Conclusion}

Highly effective and durable antibacterial PET fabrics were proposed in this work using a facile and low cost paddry-cure process. An N-halamine monomer precursor was synthesized and treated onto PET fabrics, which were rendered antibacterial activity after chlorination. The hydrophobicity of PET fabrics contributed to the antiadhesion of bacteria and offered the possibility for the persistent effect of antibacterial agents. The treated PET showed stable hydrophobicity, lasting antimicrobial property, good mechanical and thermal properties as well as rechargeable property. The produced antibacterial PET fabrics with good durability can be used as protective
A

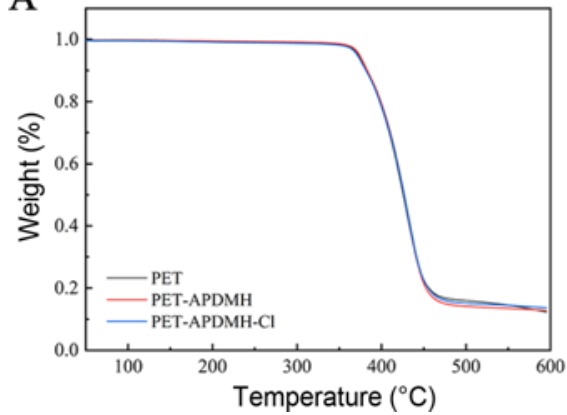

B

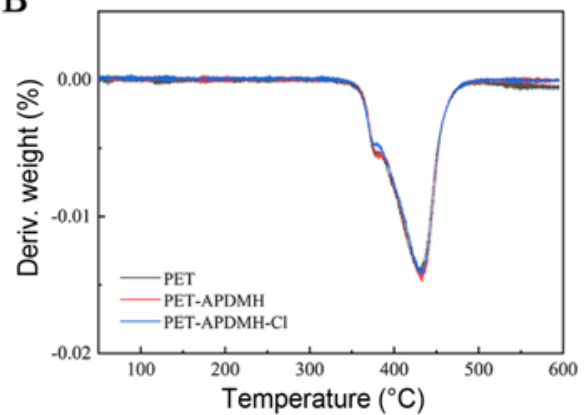

$\mathrm{C}$

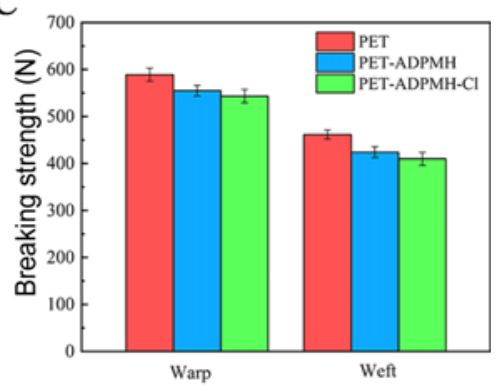

Figure 7. Thermal behaviors and mechanical properties of samples. 
clothing for working in moist and unsanitary environments.

\section{Acknowledgments}

The authors thank the funds of the Project for Jiangsu Scientific and Technological Innovation Team, the national first-class discipline program of Light Industry Technology and Engineering (LITE2018-2), and the Fundamental Research Funds for the Central Universities (NO. JUSRP52007A).

\section{References}

1. C. J. E. Metcalf and J. Lessler, Science, 357, 149 (2017).

2. Y. Zheng, N. Pan, Y. Liu, and X. Ren, Carbohyd. Polym., 253, 117205 (2021).

3. X. Yin, J. Zhang, J. Xu, M. Tian, L. Li, L. Tan, and Z. Li, Compos. Sci. Technol., 202, 108574 (2021).

4. E. D. Brown and G. D. Wright, Nature, 529, 336 (2016).

5. S. Chen, S. Zhang, M. Galluzzi, F. Li, X. Zhang, X. Yang, X. Liu, X. Cai, X. Zhu, B. Du, J. Li, and P. Huang, Chem. Eng. J., 358, 634 (2019).

6. P. Duan, Q. Xu, X. Zhang, J. Chen, W. Zheng, L. Li, J. Yang, F. Fu, H. Diao, and X. Liu, Cellulose, 27, 6603 (2020).

7. D. Gao, Y. Hou, B. Lyu, J. Ma, Y. Li, and Y. Li, Cellulose, 28, 593 (2020).

8. J. Lin, Y. Wang, X. Wei, S. Kong, Z. Liu, J. Liu, F. Zhang, S. Lin, B. Ji, Z. Zhou, and Z. Guo, Int. J. Biol. Macromol., 157, 553 (2020).

9. L. Y. Tan, L. T. Sin, S. T. Bee, C. T. Ratnam, K. K. Woo, T. T. Tee, and A. R. Rahmat, J. Vinyl. Addit. Techn., 25, E3 (2019).

10. Q. Xu, X. Ke, L. Shen, N. Ge, Y. Zhang, F. Fu, and X. Liu, Int. J. Biol. Macromol., 111, 796 (2018).

11. C. X. Wang, Y. Ren, J. C. Lv, Q. Q. Zhou, Z. P. Ma, Z. M. Qi, J. Y. Chen, G. L. Liu, D. W. Gao, Z. Q. Lu, W. Zhang, and L. M. Jin, Appl. Surf. Sci., 396, 1840 (2017).

12. M. M. Wen, I. A. Abdelwahab, R. G. Aly, and S. A. ElZahaby, Drug. Deliv., 28, 463 (2021).

13. A. A. Amiery, L Shaker, and T. Gaaz, Biointerf. Res. Appl. Chem., 11, 10393 (2020).

14. H. Xu, Z. Fang, W. Tian, Y. Wang, Q. Ye, L. Zhang, and J. Cai, Adv. Mater, 30, 1801100 (2018).

15. W. Chen, Y. Zhu, Z. Zhang, Y. Gao, W. Liu, Q. Borjihan, H. Qu, Y. Zhang, Y. Zhang, Y. J. Wang, L. Zhang, and A. Dong, Chem. Eng. J., 379, 122238 (2020).

16. Y. Gao, N. Song, W. Liu, A. Dong, Y. J. Wang, and Y. W. Yang, Macromol. Biosci., 19, e1800453 (2019).

17. L. Zhang, L. Li, L. Wang, J. Nie, and G. Ma, Appl. Surf. Sci., 515, 145962 (2020).

18. R. Wang, Y. Li, Y. Si, F. Wang, Y. Liu, Y. Ma, J. Yu, X. Yin, and B. Ding, Nanoscale. Adv., 1, 1948 (2019).

19. R. A. Baseer, G. M. Taha, A. F. Kassem, and R. Khalil,
Arab. J. Chem., 13, 5614 (2020).

20. J. Liu, C. Dong, Z. Zhang, D. Wei, and Z. Lu, Fiber. Polym., 21, 273 (2020).

21. Y. Zhao, B. Zhao, B. Wei, Y. Wei, J. Yao, H. Zhang, X. Chen, and Z. Shao, Int. J. Biol. Macromol., 165, 460 (2020).

22. S. Zhang, C. Kai, B. Liu, S. Zhang, W. Wei, X. Xu, and Z. Zhou, Cellulose, 26, 5621 (2019).

23. Y. Ma, N. Wisuthiphaet, H. Bolt, N. Nitin, Q. Zhao, D. Wang, B. Pourdeyhimi, P. Grondin, and G. Sun, ACS Biomater. Sci. Eng., 7, 2329 (2021).

24. K. Ma, Z. Xie, Q. Jiang, J. Li, R. Li, X. Ren, T. S. Huang, and K. Q. Zhang, J. Appl. Polym. Sci., 131, 40621 (2014).

25. D. Xu, X. Ma, P. Zhu, and Z. Jiang, AATCC J. Res., 7, 34 (2020).

26. D. Xu, S. Wang, J. Hu, Y. Liu, Z. Jiang, and P. Zhu, Cellulose, 28, 3265 (2021).

27. L. Qian and G. Sun, J. Appl. Polym. Sci., 89, 2418 (2003).

28. Y. Yong, M. Qiao, A. Chiu, S. Fuchs, Q. Liu, Y. Pardo, R. Worobo, Z. Liu, and M. Ma, Langmuir, 35, 1927 (2019).

29. M. Liu, F. Wang, M. Liang, Y. Si, J. Yu, and B. Ding, Compos. Commun., 17, 147 (2020).

30. F. Wang, J. Dai, L. Huang, Y. Si, J. Yu, and B. Ding, ACS Nano., 14, 8975 (2020).

31. A. Dong, Y. J. Wang, Y. Gao, T. Gao, and G. Gao, Chem. Rev., 117, 4806 (2017).

32. J. Lin, C. Winkelmann, S. D. Worley, J. Kim, C. I. Wei, U. Cho, R. M. Broughton, J. I. Santiago, and J. F. Williams, J. Appl. Polym. Sci., 85, 177 (2002).

33. X. Ren, L. Kou, H. B. Kocer, S. D. Worley, R. M. Broughton, Y. M. Tzou, and T. S. Huang, J. Biomed. Mater. Res. B., 89, 475 (2009).

34. I. Cerkez, S. D. Worley, R. M. Broughton, and T. S. Huang, Prog. Org. Coat., 76, 1082 (2013).

35. X. Ren, H. B. Kocer, L. Kou, S. D. Worley, R. M. Broughton, Y. M. Tzou, and T. S. Huang, J. Appl. Polym. Sci., 109, 2756 (2008).

36. I. Cerkez, H. B. Kocer, S. D. Worley, R. M. Broughton, and T. S. Huang, J. Appl. Polym. Sci., 133, 43088 (2016).

37. X. Li, Y. Liu, Z. Jiang, R. Li, X. Ren, and T. S. Huang, Cellulose, 22, 3609 (2015).

38. N. Pan, Y. Wang, X. Ren, T. S. Huang, and I. S. Kim, Mat. Sci. Eng. C. Mater, 103, 109877 (2019).

39. K. Bae, N. Gwak, M. K. Park, B. Lee, and K. J. Lee, Macromol. Chem. Phys., 220, 1900213 (2019).

40. Y. Liu, J. Li, L. Li, S. McFarland, X. Ren, O. Acevedo, and T. S. Huang, ACS Appl. Mater. Inter., 8, 3516 (2016).

41. Y. Zhang, M. Yin, L. Li, B. Fan, Y. Liu, R. Li, X. Ren, T. S. Huang, and I. S. Kim, Carbohyd. Polym., 243, 116461 (2020).

42. M. Zuo, N. Pan, T. S. Huang, I. S. Kim, and X. Ren, Nano., 15, 2050027 (2020).

43. Q. Kong, Z. Zhang, Z. Li, and X. Ren, Cellulose, 27, 1705 (2019).

44. M. Yin, Y. Wang, Y. Zhang, X. Ren, Y. Qiu, and T. S. 
Huang, Carbohyd. Polym., 232, 115823 (2020).

45. S. Zhang, L. Li, X. Ren, and T. S. Huang, Int. J. Biol. Macromol., 161, 1070 (2020).

46. Y. Wang, M. Yin, Z. Li, Y. Liu, X. Ren, and T. S. Huang, Colloid. Surface B., 165, 199 (2018).

47. X. Lin, X. Fan, R. Li, Z. Li, T. Ren, X. Ren, and T. S. Huang, Polym. Adv. Technol., 29, 481 (2018).

48. W. Ma, L. Li, Y. Liu, Y. Sun, I. S. Kim, and X. Ren, J. Alloy. Compound, 797, 692 (2019).

49. L. Li, K. Ma, Y. Liu, Z. Xie, T. S. Huang, and X. Ren, Polym. Adv. Technol., 25, 963 (2014).

50. W. Ma, L. Li, X. Ren, and T.-S. Huang, Cellulose, 26, 5757 (2019).

51. Q. Wang, X. Zhu, P. Zhu, and Z. Jiang, Fiber. Polym., 20, 906 (2019).

52. Y. Wang, Y. Liu, H. Tian, Y. Zhai, N. Pan, M. Yin, X. Ren, and J. Liang, Colloid. Polym. Sci., 295, 1897 (2017).

53. Y. Chen, C. Feng, Q. Zhang, G. Ren, and Q. Han, Appl. Surf. Sci., 467, 526 (2019).

54. X. Tang, H. Xu, Y. Shi, M. Wu, H. Tian, and J. Liang, Carbohyd. Polym., 229, 115546 (2020).

55. Y. N. Gao, Y. Wang, T. N. Yue, Y. X. Weng, and M. Wang,
J. Colloid. Interface. Sci., 582, 112 (2021).

56. L. Rong, H. Liu, B. Wang, Z. Mao, H. Xu, L. Zhang, Y. Zhong, X. Feng, and X. Sui, Carbohyd. Polym., 211, 173 (2019).

57. J. Lin, X. Chen, C. Chen, J. Hu, C. Zhou, X. Cai, W. Wang, C. Zheng, P. Zhang, J. Cheng, Z. Guo, and H. Liu, ACS Appl. Mater. Inter., 10, 6124 (2018).

58. S. Zhang, F. Ding, Y. Wang, X. Ren, and T. S. Huang, Fiber. Polym., 21, 1023 (2020).

59. Y. Chen, P. Yu, C. Feng, Y. Wang, Q. Han, and Q. Zhang, Appl. Surf. Sci., 419, 683 (2017).

60. Y. Chen, Q. Chen, Y. Wang, Q. Zhang, and Q. Han, Appl. Surf. Sci., 535, 147702 (2021).

61. M. Chylinska, H. Kaczmarek, and A. Burkowska-But, Colloid. Surface B., 176, 379 (2019).

62. Y. Y. Xie, X. H. Hu, Y. W. Zhang, F. Wahid, L. Q. Chu, S. R. Jia, and C. Zhong, Carbohyd. Polym., 229, 115456 (2020).

63. S. Hameed, Y. Wang, L. Zhao, L. Xie, and Y. Ying, Mat. Sci. Eng. C. Mater, 108, 110338 (2020).

64. T. Mu, N. Pan, Y. Wang, X. Ren, and T.-S. Huang, Fiber. Polym., 19, 2284 (2018). 